\title{
PNEUMATIC STRETCHER-CHAIR DEVICE FOR PARALYSED PATIENTS
}

\author{
Padmanabhan $M^{1}$, Rahoof $\mathrm{T} \mathrm{E}^{2}$, Vipin Raj V $\mathbf{M}^{3}$, Vivek Krishnan $\mathrm{K}^{\mathbf{4}}$ \\ ${ }^{I}$ Student, Mechanical Engineering Department, MES College of Engineering, Kuttippuram, Kerala, India \\ ${ }^{2}$ Student, Mechanical Engineering Department, MES College of Engineering, Kuttippuram, Kerala, India \\ ${ }^{3}$ Student, Mechanical Engineering Department, MES College of Engineering, Kuttippuram, Kerala, India \\ ${ }^{4}$ Student, Mechanical Engineering Department, MES College of Engineering, Kuttippuram, Kerala, India
}

\begin{abstract}
In this age of nuclear families, the smaller number of available caregivers for paralyzed patients is a major problem. The shifting of such patients to a wheelchair is a delicate process and in most of the instances, two or more caregivers are required. This paper presents the design and fabrication of pneumatically powered stretcher-chair convertible device with movable support segments in an attempt to help such patients and caregivers. This helps the caregiver avoid heavy lifting situations that put their back at risk of injury. The caregiver can merely shift the patient from a bed on to the device while the device is in the form of a stretcher. Then the device can be converted into a chair and the wheels of the device provide mobility like a wheelchair. Most paralyzed patients develop pressure ulcers which add to their misery causing immense pain and irritation. Support surface pressure or the reaction pressure of the surface on which the patient's body is supported has been identified as a major cause of pressure ulcer formation. In order to address this scenario, an idea of alternating pressure support surface has been implemented. This mechanism relieves support surface pressure on the patient's body thus preventing/ prolonging pressure ulcer formation. It also exposes the body to more air so that perspiration on the patient's skin evaporates better. Further, this mechanism acts as a body/ muscle stimulating mechanism. The alternating pressure support surface is also actuated by pneumatic power and controlled by an electronic timing circuit.
\end{abstract}

Keywords: Paralysis, Pressure ulcers, Pneumatics, Stretcher-chair, alternating pressure surface

$* * *$

\section{INTRODUCTION}

Innovations from the field of engineering have revolutionized the arena of medical sciences significantly during the last few decades. Engineering sciences have come up with numerous aids for the patients. Out of these, mechanical aids for physically impaired, especially for immobile patients are a major class. Assistive technology for the mobility of impaired includes the wheelchair, lift aids and other devices, all of which have been around for centuries.

\subsection{Paralysis}

Paralysis is a medical condition in which the muscle loses its capacity to function in a part of the body making that part immobile [5]. Basically, paralysis happens when there is some sort of nerve damage, that is, either there is a problem with any nerve or when a portion of the spinal cord is injured. The medical condition Motor Neuron Disease (MND) needs to be mentioned here. It describes a group of related diseases, affecting the motor nerves or neurons in the brain and spinal cord, which pass messages to the muscles telling them what to do. This disease often causes paralysis. This progressive neuro-degenerative disease attacks the upper and lower motor neurons, leading to weakness and wasting of muscles.

\subsection{Pressure Ulcers}

Pressure ulcers (generally called bed sores) are areas of damage to the skin and underlying tissue [7]. Pressure ulcers may develop very quickly in some individuals if they are immobile even for a very short time. As such, paralyzed patients are the easiest victims of pressure ulcers. Pressure ulcers can be very serious and painful. They can damage not just the skin, but also deeper layers of tissue under the skin. One of the best ways of preventing pressure ulcers is to avoid heavy and constant pressure on vulnerable parts of the body. Proper air circulation of air over the body is also of prime importance from the perspective of pressure ulcer prevention.

\section{PROBLEM IDENTIFICATION}

Paralysis patients are highly dependent on their caregivers. Today, number of available caregivers per patient is showing a decreasing trend. The transfer of these immobile patients from bed to a wheel chair is a delicate process and in most of the instances, two or more caregivers are required. However, it is estimated that 1 in 3 nurses or caregivers will develop back injuries. Most injuries occur because the patient is relatively heavy to lift and access to them is difficult when attempting to place the patient onto another seat. This poses a need for improving the available support devices to ease the effort of 
available caregivers. Besides this, the inconvenience to the patient during the transfer procedure also needs to be addressed. The transfer procedure gets harder with increase in the patient weight and with the degree of immobility. Spending long durations on bed lead to development of bedsores in most patients. This causes tremendous pain and irritation. The compressions of skin under pressure for long durations have been identified as one of the causes for this. Also the microclimate (temperature, humidity etc.) around the skin is a parameter that affects bedsore formation.

After some case studies, a device which proves to be an apt solution to the problem described above was developed. This paper describes the design and fabrication of this device.

\section{LITERATURE REVIEW}

\subsection{Patents Referred}

In January 1988, a convertible bed and wheelchair unit was patented by Willis G. Shaffer ${ }^{[1]}$. It comprised of a wheeled structure which was readily convertible between a full-sized single bed and a wheelchair. However this conversion proved to be difficult when a patient occupied the device.

In August 1997, Norman Weiss ${ }^{[2]}$ invented wheelchair and bed with movable body supporting portions. This was a wheel chair/bed for paralyzed invalids comprises a support on which the invalid rests, the support comprising a plurality of support segments which define a support surface. The support segments can be moved simultaneously and act as a pressure relieving mechanism.

\subsection{Researches on Pressure Ulcers ${ }^{[7]}$}

In 1959, Kosiak reported a relationship between amount of pressure, duration of application and the development of tissue damage in canine and rat experiments. In the 1970s, Reswick and Rogers published guidelines based on human observations that depicted non-injurious and injurious levels and lengths of exposure to particular interface. In 1995, Kokate et al and Iaizzo et al, by their experiments on pigs concluded that skin and soft tissue damage due to pressure could be reduced by localized skin cooling. In 2009, it has been proposed that high pressures can cause pressure damage within a relatively short time, but that lower pressures can be applied for long periods without damage occurring. The studies by Brown $\mathrm{M}$ et al in 1981 and Oertwich P A et al in 1995 revealed the impact of making small, frequent movements of a patient's body slightly with each contact. In an experiment to measure the effects of pressure on blood flow in the human forearm, Shitamichi M et al in 2009 concluded that blood flow was affected by pressure on the skin to a greater extent in a deep artery than in a skin capillary.

\section{DESIGN AND PRINCIPLES ${ }^{[4,6]}$}

a)
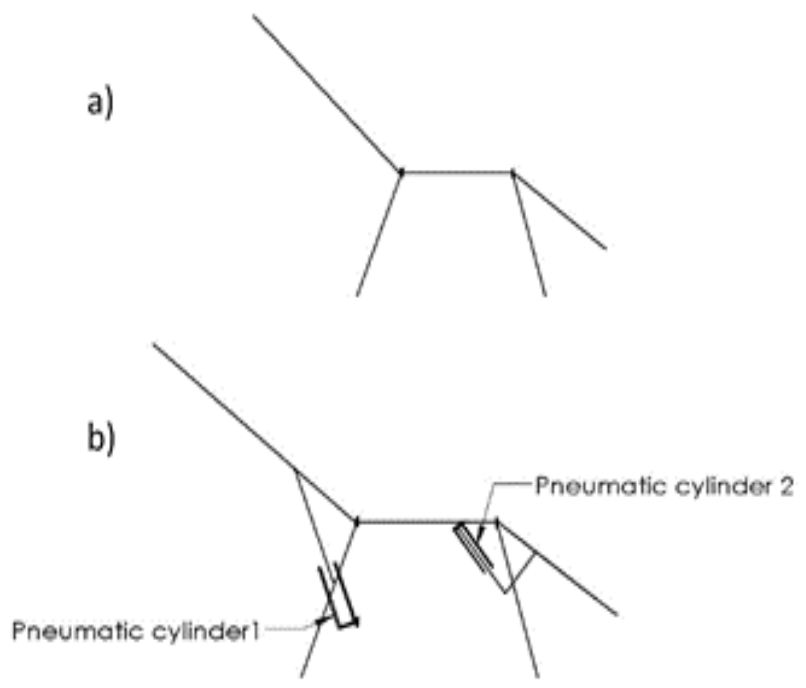

Fig 1: Concept generation

The basic design of the device has evolved from the concept of a convertible wheelchair. Thus the initial sketches for the project design included only the skeletal structure of a wheelchair (Fig:1.a). Then the convertible feature was included to the design at a conceptual level and tremendous amount of brainstorming was done. Since the fundamental aim of is to provide comfort to the patient as well as his/ her caregiver, powered conversion of the device between chair and stretcher was given focus.

In the second phase, the power source was to be selected. Though hydraulic actuators are the best in terms of load handling and stability, the relatively high cost and the bulkiness of the units hindered their choice for our purpose. Electric motors have good load carrying capacity, speed control characteristics, precision etc. But their implementation necessitates the use of gears, belts or pulleys making the over unit becomes bulky and heavy. So we go for pneumatic power ((Fig:1.b) where simple actuators like the pneumatic cylinder are the only entities that are to be fixed onto the device. The compressed air supply could be some distance away from the device. Pneumatic actuators provide high force and speed at low unit cost in a small footprint. As a matter of fact, pneumatic cylinders provide more force and speed per unit size than any other actuator technology except hydraulic. Force and speed on pneumatic actuators are easily adjustable and are independent of each other.

The next phase of design was the choice of pneumatic cylinder specifications and the actuating mechanism as well as the link manipulation. After considering various space and convenience factors, inverted-slider crank mechanism was selected for pneumatic cylinders $1 \& 2$. Further the layout was 
drawn using SolidWorks 11 and detailed examination of link dimensions, cylinder-link configurations, was done using MS Excel on a trial and error basis. Once the design of links is fixed, similar trial and error procedure was repeated for various cylinder pressure, piston force and link configurations, incorporating the load/ the patient weight on the device. Since the design and fabrication of a prototype was intended, a maximum patient weight of $50 \mathrm{~kg}$ was set.

The patient's body weight supported by each section of the device was estimated as per the percentage body weight distribution suggested in the book, Human Body Dynamics: Classical Mechanics and Human Movement by Aydin Tozeren [3]. Also, based on wheelchair ergonomics and posture analysis, the optimum angle between upper body and thigh region while sitting is $100-110^{\circ}$ angle at the knee joint (between thighs and lower legs) is $105-110^{\circ}$.

\subsection{Formulations Used}

The formulations used have been briefly explained here. The results corresponding the fabricated prototype (discussed in subsequent section) for a patient weight $50 \mathrm{~kg}$ is also mentioned briefly.

\subsubsection{Pneumatic Cylinder- 1}

Let $\mathrm{W}_{1}$ be the total load corresponding to head section which is algebraic sum of weight of head section in empty condition and patient's body weight on the head section. Let the centre of gravity of the head section lies at a distance $a_{1}$ from the corresponding hinge joint.

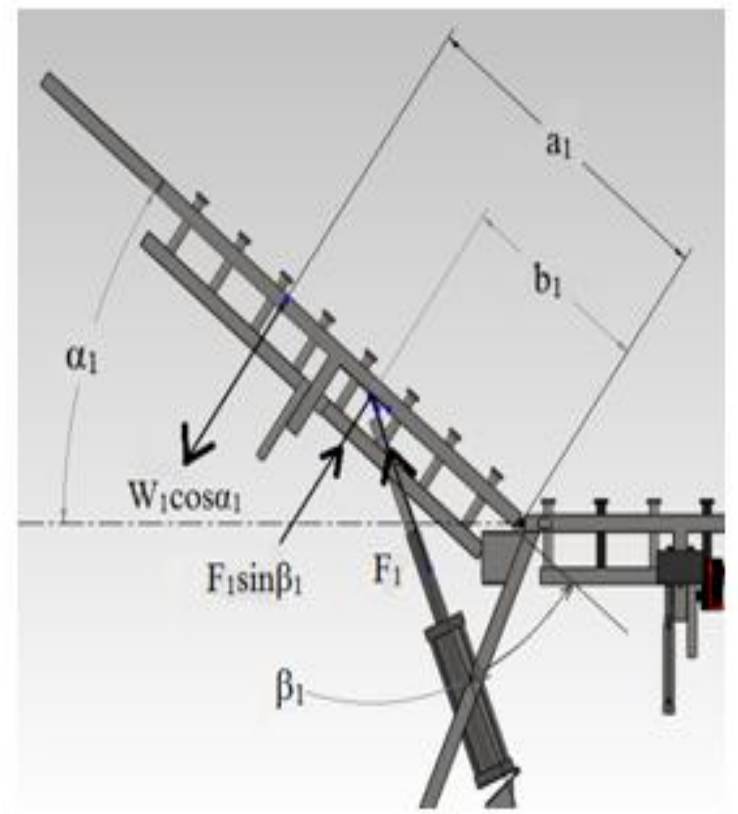

Fig 2: Formulations for pneumonic cylinder 2
The moment of head section about the hinge,

$$
M_{1}=W_{1} \cos \alpha_{1} \times g \times a_{1}
$$

Let $\mathrm{F}_{1}$ be the piston force of the pneumatic cylinder-1.

For the outstroke of a double acting cylinder, the force is calculated as

$$
F_{1}=P_{1} \times \frac{\pi d_{1}^{2}}{4}
$$

Where

$\mathrm{P}_{1}=$ Pressure acting on the cylinder piston

$\mathrm{d}_{1}=$ bore of the cylinder

This force acts along a hinged link as shown in figure. As in the figure, this force acts at a distance of $b_{1}$ from the hinge of head section.

The torque due to force $\mathrm{F}$ which counters the moment $\mathrm{M}_{1}$ is

$$
T_{1}=F_{1} \sin \beta_{1} \times b_{1}
$$

Now, net torque

$$
T_{\text {net }-1}=T-M_{1}
$$

The angles $\alpha_{1}$ and $\beta_{1}$ vary with change in one another and their variations were obtained by geometrical and statistical methods.

For a patient weighing $50 \mathrm{~kg}$, the load on head section including trunk, head, neck, forearms and hand of the patient totals to about $30 \mathrm{~kg}$. Also empty weight of head section of the prototype $=10 \mathrm{~kg}$.

$$
\therefore \quad W_{1}=30+10=40 \mathrm{~kg}
$$

Calculations favored the choice of $50 \mathrm{~mm}$ cylinder bore operating at 5 bar pressure which delivers an average net torque of 12167.03 N.mm From the standard pneumatic cylinders available, a cylinder $50 \mathrm{~mm}$ diameter and $100 \mathrm{~mm}$ stroke length was selected.

\subsubsection{Pneumatic Cylinder- 2}

Let $\mathrm{W}_{2}$ be the total load corresponding to head section which is algebraic sum of weight of foot section in empty condition and patient's body weight on the foot section. Let the centre of gravity of the head section lies at a distance $a_{2}$ from the corresponding hinge joint. 


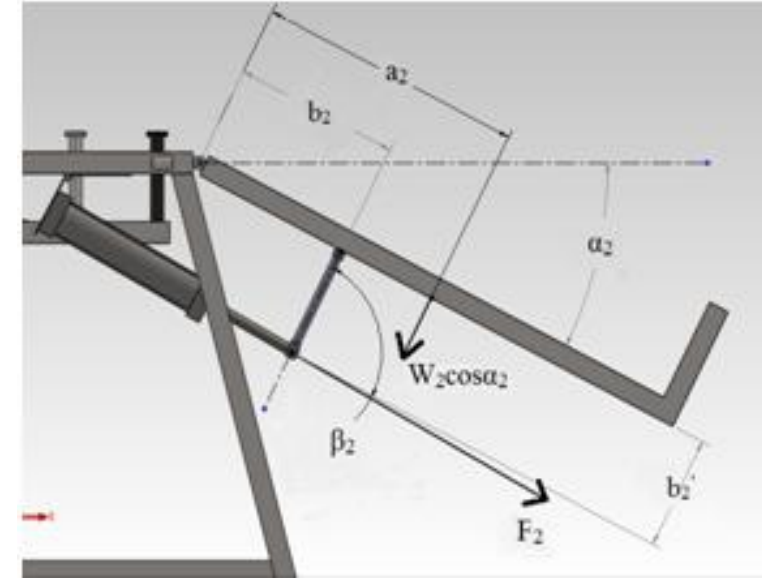

Fig 3: Formulations for pneumonic cylinder 2

The moment exerted by load $\mathrm{W}_{2}$ on the foot section hinge,

$$
M_{2}=W_{2} \cos \alpha_{2} \times g \times a_{2}-(5)
$$

Let $\mathrm{F}_{2}$ be the piston force of the pneumatic cylinder- 2 .

For the outstroke of a double acting cylinder, the force is calculated as

$$
F_{2}=P_{2} \times \frac{\pi d_{2}^{2}}{4}
$$

Where $\mathrm{P}_{2}=$ Pressure acting on the cylinder piston

$$
\mathrm{d}_{2}=\text { bore of the cylinder }
$$

As implied by the figure, the force $F_{2}$ has a component perpendicular to the foot section and one parallel to it, whose lines of action are both displaced over some distance from hinge.

Thus there are two resulting torques:

$$
\begin{aligned}
& T_{\uparrow}=F_{2} \cos \beta_{2} \times b_{2} \\
& T_{\rightarrow}=F_{2} \sin \beta_{2} \times b_{2}^{\prime}
\end{aligned}
$$

The resulting torque is given by

$$
T_{2}=T_{\uparrow}+T_{\rightarrow}
$$

Then net torque on foot section hinge is

$$
T_{\text {net }-2}=T_{2}-M_{2}
$$

The angles $\alpha_{2}$ and $\beta_{2}$ vary with change in one another and their variations were obtained by geometrical and statistical methods.
For the prototype, empty weight of foot section is $3 \mathrm{~kg}$ and from human anatomical studies, the weight of the patient (whose total weight is $50 \mathrm{~kg}$ ) that the foot section supports is $6.8 \mathrm{~kg}$.

$$
\therefore W_{2}=6.8+3=9.8 \sim 10 \mathrm{~kg}
$$

Calculations favored the choice of $25 \mathrm{~mm}$ cylinder bore. In the course of calculation, positive torque was obtained from pressure 4 bar onwards. Since 5 bar operating pressure was consistent for pneumatic cylinder- 1 , the same shall be applied here. The average net torque obtained at 5 bar pressure and 25 $\mathrm{mm}$ cylinder bore is 22368.43 N.mm. From the standard pneumatic cylinders available, a cylinder $25 \mathrm{~mm}$ diameter and $80 \mathrm{~mm}$ stroke length is selected.

\section{FABRICATION OF PROTOTYPE}

The fabricated model has the following main components: framework movable support segments and pneumatic components which are discussed below.

\subsection{Framework}

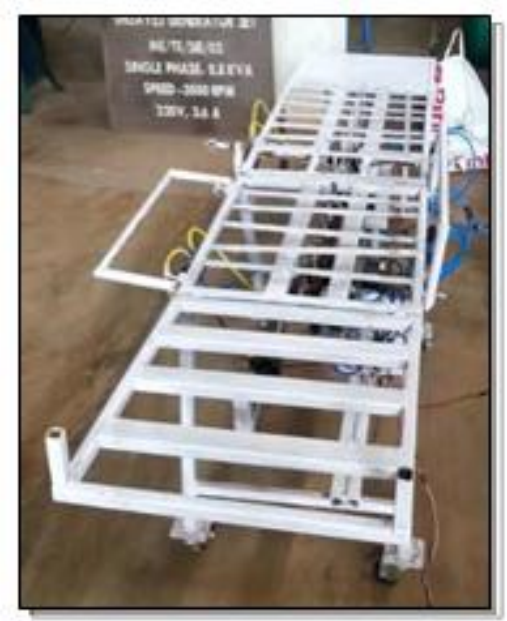

Fig 2: The framework with movable segments laid on it

The entire fabrication of the device has been done around a basic framework. The framework has been made by welding together mild steel pipes of square and rectangular cross sections. The relative low cost, availability and properties like decent tensile, compressive strengths and weldability favored the choice of mild steel over other metals. The frame has three main sections, namely the head, seat and foot sections. The framework along with the support surfaces bear the patients weight. The seat section forms the basis as far as the assembling is concerned. The head section and foot section are hinged to the seat section. The movable support segments (Refer Section IV.B) are anchored in the seat and head sections. The movable segments are then given a cushion 
each. Also the head section is give a pillow type cushion. Arm rests are attached on either sides of seat section such that they can be swung sideways with the seat edge as the pivot. The legs of the fabricated prototype is integral to the seat section. The angular orientation of the legs are so as to provide the best balance. Each leg terminate into a flange on which a wheel is attached. Wheels of $150 \mathrm{~mm}$ diameter have been used here. The wheels provide the mobility to the device both in the stretcher and chair position. Each wheel has the ability to facilitate steering.

\subsection{Movable Supporting Segments}

The support surface on which patient sits/lies is multisegmented and movable. There are two groups of movable segments each which actuate the alternating pressure support surface in the head and seat sections. The skeletal structures of the movable segments have been fabricated by welded M.S. flat and circular M.S. rods. The movable segments are anchored by certain cylindrical holes in the head and seat sections and constrain their motion to that of a slider. The support segments in both head and seat sections can be identified as two sets. In assembly, the two sets are placed as though they appear to be sandwiched between one another.

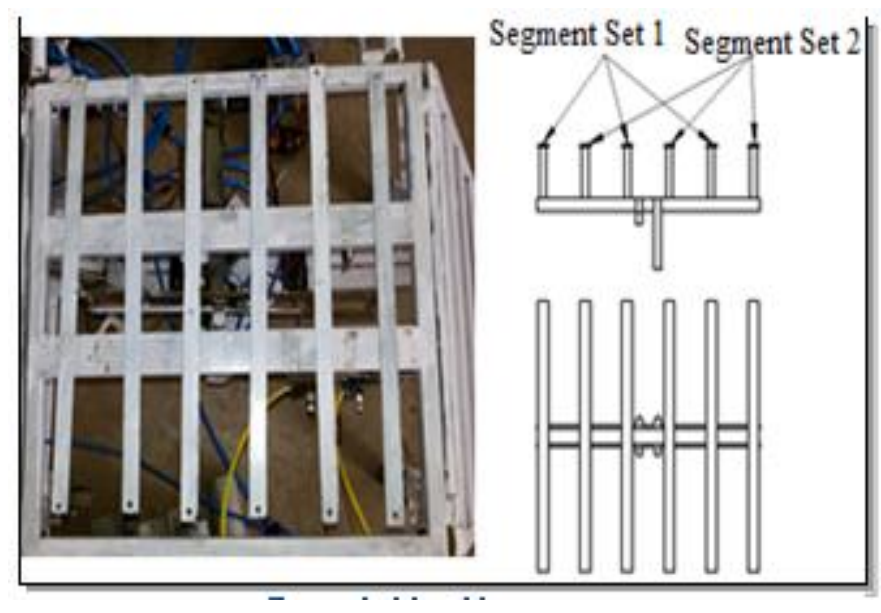

Fig 3: Movable segments

\subsection{Pneumatic Components}

The power for the stretcher-chair conversion is pneumatic, as mentioned in the design section. The pressure energy of compressed air is converted into mechanical force by pneumatic cylinders. Double acting cylinders of three different specifications have been used depending on the load and orientation they are subjected to. The three cylinders are labelled as cylinder-1, cylinder- 2 and cylinder- 3 . Pneumatic cylinder-1 powers the stretcher chair conversion of the head section. This cylinder is fixed on a hinge and is capable of executing an oscillatory motion. The piston rod of this cylinder terminates in a hinged joint on the head section. Pneumatic cylinder-2 is responsible for powering the conversion process in foot section. This cylinder is also has its base fixed on a hinge and can oscillate about this hinged joint. Pneumatic cylinder- 3 executes the alternating motion of the movable segments. Each set of segment in head and seat sections has a cylinder each associated with them.

Specifications of pneumatic cylinders used:

\begin{tabular}{|l|l|l|l|}
\hline $\begin{array}{l}\text { Pneumatic } \\
\text { cylinder: }\end{array}$ & 1 & 2 & 3 \\
\hline Make & Gavantech & Gavantech & Gavantech \\
\hline Bore & $50 \mathrm{~mm}$ & $25 \mathrm{~mm}$ & $16 \mathrm{~mm}$ \\
\hline Stroke & $100 \mathrm{~mm}$ & $80 \mathrm{~mm}$ & $50 \mathrm{~mm}$ \\
\hline $\begin{array}{l}\text { Pressure } \\
\text { range }\end{array}$ & $3-10 \mathrm{bar}$ & $3-10 \mathrm{bar}$ & $3-10 \mathrm{bar}$ \\
\hline No:s & 1 & 1 & 4 \\
\hline
\end{tabular}

The solenoid valves control the timing of cylinder activation by regulating the flow of compressed air into the cylinders. The solenoid valves used are $5 / 2$, i.e. 5 ports and 2 switching positions. Pneumatic tubes convey the compressed air from a storage tank to the pneumatic cylinders which actuate all the movements in the fabricated device.

Specifications of solenoid valves:

\begin{tabular}{|l|l|}
\hline Make & Akari \\
\hline Electrical specifications & $220 \mathrm{~V} \mathrm{AC}, 6.0 \mathrm{VA}, 2.9 \mathrm{~mA}$ \\
\hline No:s & 4 \\
\hline
\end{tabular}

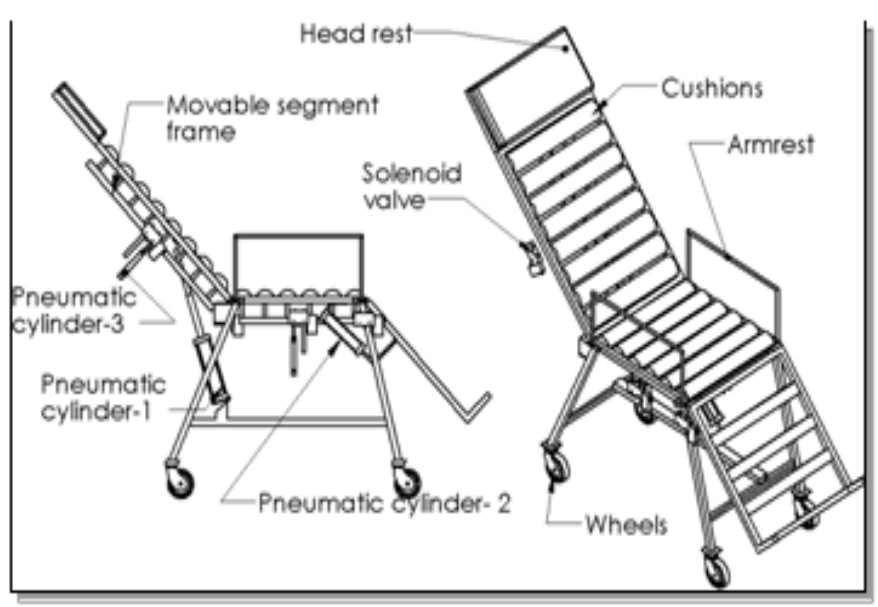

Fig 4: Assembled views of prototype

\section{WORKING OF THE DEVICE}

The stretcher chair device that was designed and fabricated does two main operations described below. 


\subsection{Stretcher- Chair Conversion}

The framework represents the bed-chair convertible unit which act as the chassis on which all other components are assembled.

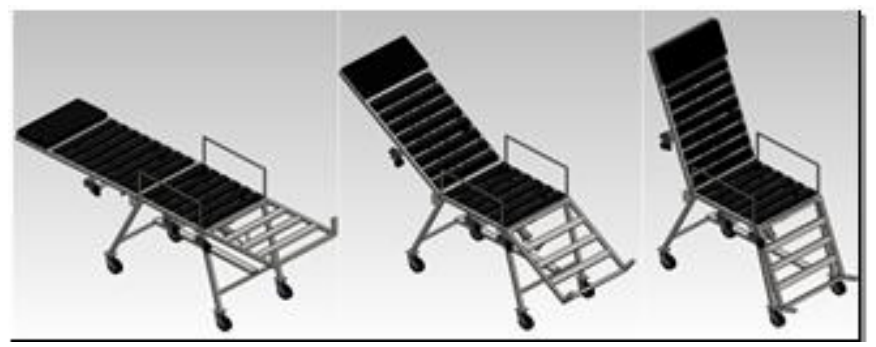

Fig 5: Stretcher-chair conversion

In the stretcher forming position of the unit, the three sections viz. head, seat and foot sections are disposed horizontally to form a continuous mattress support surface. The auxiliary legwheel unit is deployed for providing support to the head section. The sections can be secured in position by locking mechanisms. In this position, the pneumatic cylinder-1 is in inward stroke and the pneumatic cylinder-2 is in outward stroke. Whenever the chair forming position is desired, a switch is used to make the pneumatic cylinder-1 execute an outstroke and pneumatic cylinder-2 an inward stroke. As a result the head section is swung upwardly, while the foot section swings down. During this conversion the head section and foot section both sweeps an angle of $70^{\circ}$. Thus, the positions can be changed to stretcher or chair as required, by controlling the inward and outward strokes of pneumatic cylinders.

\subsubsection{Patient Transfer}

The stretcher- chair conversion is intended to assist patient transfer. The following procedure is recommended for patient transfer from bed to our device:

Bring the device to the stretcher position by using control switches. Swing the armrests down and bring the device sideby-side with the patient's bed. Transfer the patient onto the device by simply dragging the patient over the bed sheet. Devices such as patient shifter can make this transfer simpler. Move the stretcher away from the bed and bring back the armrests to initial position. Using the control switches, this stretcher can be converted into a wheelchair or else the alternating pressure system using movable segments can be activated.

\subsection{Alternating Pressure Surface}

The movable body supporting members of the device make it an alternating pressure surface. Alternating pressure support surface is intended to ultimately reduce chances of pressure ulcer formation. Alternating pressure support surfaces redistribute pressure on the patient's body by cyclically moving up and down at alternate zones of the surface.

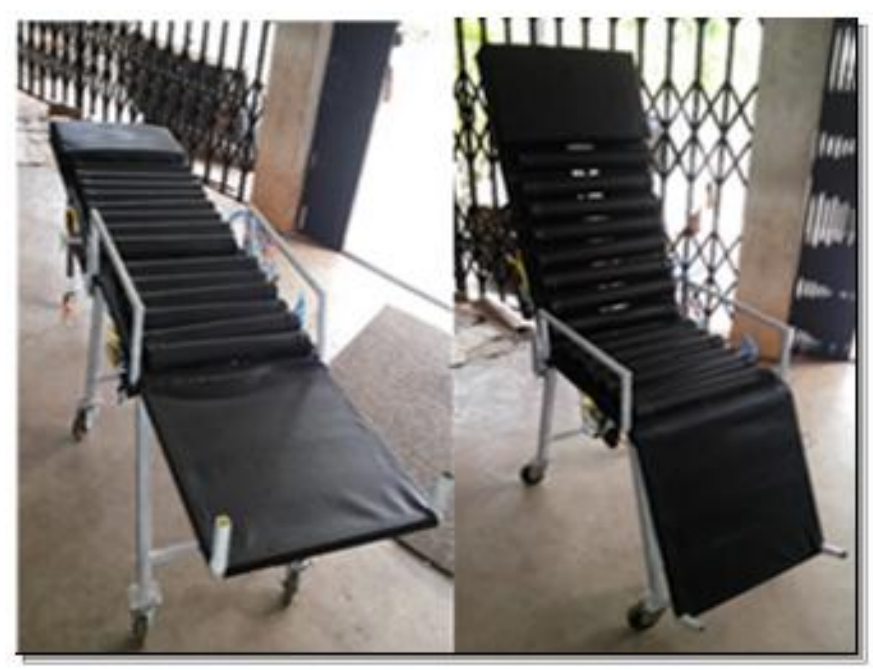

Fig 6: The fabricated prototype

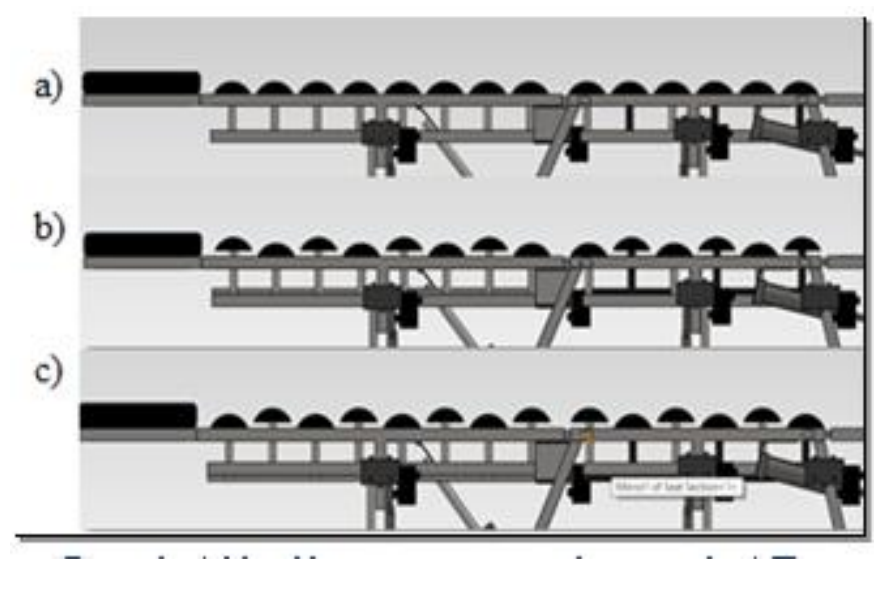

Fig 7: a) Movable segments in normal position, (b, c) The alternate motion of segments

As mentioned before, the support surface of the device is segmented and forms two sets each in head and seat sections. The supports in each set are movable in such a way that each mattress segment in a group can be moved simultaneously into and out of engagement with the body of a patient. As one group of mattress segments moves up the other is taken out of engagement with the body of a patient. Similarly, as the segments in second set moves up the first set moves down there by disengaging from patient's body. Due to the alternating motion of support segments, the parts of the skin which were in contact with some support segments are relieved from the support surface pressure, while other parts of the skin of the patient are engaged and supported by support segments. It further exposes body to air so that perspiration on that portion of the skin can evaporate. 
Pneumatic cylinders- 3 are responsible for the alternating pressure surface function. The four cylinders are connected to four sets of movable segments, two each in head and seat sections. The outstroke of these cylinders give an upward motion to the associated segments. During instroke, the cylinder piston moves down and the segments come down to their normal position. A timer circuit consisting of a timer, an IC and relay controls the change in cylinder activation. Say the duration of a single cycle is 2 seconds. Then, during the first segment the timer circuit activates one set of cylinders each in head and seat sections for the 1st second. During 2nd second, the next set of cylinders are activated while other cylinders execute instroke. The timer circuit simply controls action of solenoid valves. The solenoid valves thus supply air to the cylinders in an alternating manner.

\section{CONCLUSIONS}

A prototype of the pneumatic stretcher-chair device with an alternating pressure support surface for a patient weight of $50 \mathrm{~kg}$ was designed and fabricated. The chair to bed conversion feature of this device makes patient transfer easier. The use of simple shifting aids such as patient shifters can further optimize the patient transfer by avoiding heavy lifting situations and possible back injury to the caregivers. This device combines the concept of patient mobility, patient transfer as well as pressure ulcer prevention. Literature about such a combination is scarce. This enables the user to have two fold utility and satisfaction. Thus the spending is reduced. The mean time to develop a pressure ulcer is prolonged by over 20 days than on conventional devices or support surfaces. The pneumatic components used herein have simple operation principles. Therefore, fault detections are simple. Also the pneumatic system proves to be non-hazardous.

Though the advantages of the fabricated device are multifold, there is still room for improvement as far as fabrication is concerned. The first improvement suggested is the use of electronic systems to perfectly synchronize the motion of head and foot section during a conversion. The head section tends to jerk at the beginning of a chair to bed conversion which must be solved preferably by a damper. The system pressure variations also affect the device's performance.

\section{SCOPE FOR FUTURE WORK}

Designing ergonomic cushions for the support section comprising of movable segments implemented herein can further reduce pressure ulcer formation. Such a work shall also address other pressure ulcer causing factors like friction and shear. The ideal frequency, duration and amplitude of the segment motion need to be determined. There is no literature available on this matter. Experiments and careful examination with the aid of medical experts is recommended for deriving the optimum values of these parameters. A pneumatic duct system that circulates the air exhausted from the cylinders such that it passes over patients back is highly recommended.
This would further improve the evaporation of sweat from the invalid's body.

\section{ACKNOWLEDGMENTS}

The authors express their deepest sense of gratitude to $\mathrm{Mr}$. Shine K, Asst. Professor, Mechanical Engineering Department, MES College of engineering, Kuttippuram, Kerala, who has been the guide and the source of motivation during for this work. The authors also express their indebtedness to Mr. Varun Raj K P, student, MES College of Engineering, Kuttippuram for being part of this work. The authors also thank entire staff of Mechanical Engineering Department, MES College of engineering who have supported and encouraged their work.

\section{REFERENCES}

[1] Willis G. Shaffer, "Convertible bed and wheelchair", US Patent Number: 4,717,169, 1988

[2] Norman Weiss, "Wheelchair and bed with movable body supporting portions", US Patent Number: 5,659,910, 1997

[3] Jan Dul and Bernard Weerdmeester, "Ergonomics for Beginners", $2^{\text {nd }}$ edition., Taylor \& Francis Inc., 2001

[4] P Croser, F Ebel, "Pneumatics: A Basic Level Textbook", Festo Didactic GmbH \& Co, Denkendorf, 2002

[5] Roger Bostelman and James Albus, "Robotic patient lift and transfer", Service Robot Applications, InTech, 2008

[6] S S Rattan, "Theory of Machines", $3^{\text {rd }}$ edition, Tata McGraw Hill Education Pvt. Ltd., 2009

[7] H L Orsted et al., "International review: Pressure ulcer prevention: pressure, shear, friction and microclimate in context- A consensus document", London: Wounds International, 2010.

\section{BIOGRAPHIES}

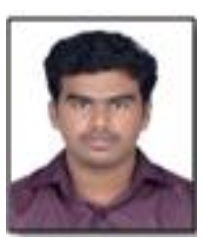

Padmanabhan $\mathbf{M}$ is currently pursuing his B-Tech degree in Mechanical Engineering from MES College of Engineering, Kuttippuram, Kerala. His areas of interest include Thermal Engineering, Industrial Engineering and Computer Aided Design. He is ambitious and aspires to be part of quality research ventures.

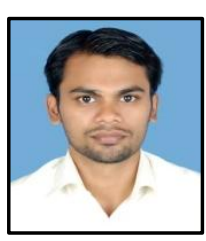

Rahoof T E is currently pursuing his B-Tech degree in Mechanical Engineering from MES College of Engineering, Kuttippuram, Kerala. He has a great passion for Automobile Engineering and dreams about a career in the Automobile industry. 


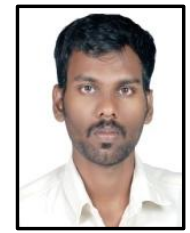

Vipin Raj V $\mathbf{M}$ is currently pursuing his BTech degree in Mechanical Engineering from MES College of Engineering, Kuttippuram, Kerala. He has a keen interest in Machine Design and Solid Mechanics. He is committed theoretical arena.

to do experimental analysis beyond the

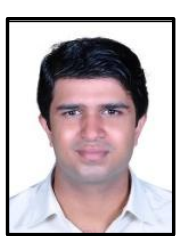

Vivek Krishnan $\mathbf{K}$ is currently pursuing his BTech degree in Mechanical Engineering from MES College of Engineering, Kuttippuram, Kerala. He is passionate about Computer Aided Manufacturing systems. He has also a keen interest to Operations Research and favours the application of numerical methods to engineering problems. 